\title{
Propagation and reflection properties of magnetoelastic wave in FeSiB amorphous wire
}

\author{
Y. Takemura, S. Masuda, ${ }^{\text {a) }}$ T. Yamada, and K. Kakuno \\ Division of Electrical \& Computer Engineering, Yokohama National University, 156 Tokiwadai, Hodogaya- \\ ku, Yokohama 240, Japan
}

Propagation and reflection properties of magnetoelastic waves in $\mathrm{Fe}_{77.5} \mathrm{Si}_{7.5} \mathrm{~B}_{15}$ amorphous wires are studied. The attenuation constant increases with increasing the driving frequency of the wave. As the attenuation constant of the wave driving at $500 \mathrm{kHz}$ is quite small $\left(0.18 \mathrm{~m}^{-1}\right)$, the wave is expected to be observed after the propagation of 20 or $30 \mathrm{~m}$. As for the reflection, the magnetoelastic wave is reflected with the efficiency as much as 0.9 at the driving frequency below $2 \mathrm{MHz}$ using a clamp as reflector. (c) 1996 American Institute of Physics. [S0021-8979(96)65908-2]

\section{INTRODUCTION}

Amorphous magnetic wires, such as $\mathrm{FeSiB}$ and $\mathrm{FeCoSiB}$ wires, provide attractive features including the magnetoelastic wave ${ }^{1}$ and the magnetoimpedance effect. ${ }^{2}$ Among them, it has been found that the magnetoelastic wave in $\mathrm{FeSiB}$ amorphous wire is well controllable and that it is suitable for sensors and the other applications. A large magnetostriction $\left(\lambda_{s}=35 \times 10^{-6}\right)$ in $\mathrm{FeSiB}$ realizes a large magnetoelastic coupling, and an efficient driving and pickup of the magnetoelastic wave. Due to the high electrical resistivity of amorphous $\mathrm{FeSiB}$, an eddy current loss of the propagated magnetoelastic wave is low. Only a longitudinal mode of the magnetoelastic wave is propagated in the onedimensional shape of the wires. Because of these characteristics, the attenuation constant of the magnetoelastic wave is low $\left(0.18 \mathrm{~m}^{-1}\right.$ at $\left.500 \mathrm{kHz}\right)$ and it is propagated through the long distance, which is applicable for the wide-range distance sensor. ${ }^{3}$ It detects a distance from 0 to $5 \mathrm{~m}$ with an accuracy less than $1 \mathrm{~mm}$. In the sensor, sensitivity and resolution depend much on an attenuation constant and a reflection efficiency of the wave at the reflector. The experimental study of the propagation and reflection properties of magnetoelastic waves in $\mathrm{FeSiB}$ wire are reported.

\section{EXPERIMENTS}

Amorphous $\mathrm{Fe}_{77.5} \mathrm{Si}_{7.5} \mathrm{~B}_{15}$ wires supplied from Unitika Co., Japan are used in this study. They are produced by the in-rotating-liquid spinning method. ${ }^{4}$ As-quenched wires of $125 \mu \mathrm{m}$ in diameter are cold drawn to $90 \mu \mathrm{m}$. The as-drawn wires are magnetically hard. They have the following features: (1) a low efficiency to drive or to pick up the magnetoelastic wave, (2) a low attenuation of the magnetoelastic wave, (3) few interaction between the magnetoelastic wave and external magnetic and electric fields.

In order to realize the efficiency driving and pickup of the wave, small regions of the as-drawn wire are annealed by the partial annealing technique. ${ }^{1}$ The annealing is performed by blowing a hot air from a nozzle connected with a ceramic heater $\left(400{ }^{\circ} \mathrm{C}\right.$ for $15 \mathrm{~min}$ without applying magnetic field). The annealed parts are magnetically soft and they are settled

\footnotetext{
${ }^{a}$ Research \& Development Dept., Jeco Co. Ltd., 1-4-1 Fujimicho, Gyodashi, Saitama 361, Japan.
}

at the positions of driving and pick-up coils as shown in Fig. 1. Bias coils surrounding the driving and pick-up coils are equipped in order to enhance an efficiency in driving and pickup of the wave. By applying a high-frequency current through the driving coil, the magnetoelastic wave is excited in the wire. The magnetoelastic wave excited by the highfrequency current of a tone-burst waveform is used for measurements of amplitudes of the wave. Details on driving and pickup of the wave have been reported. ${ }^{1}$ The velocity of the wave is $4.7 \mathrm{~km} / \mathrm{s}$ and its wavelength is about $1 \mathrm{~mm}$ at the driving frequency of $5 \mathrm{MHz}$.

\section{RESULTS AND DISCUSSION}

\section{A. Propagation property}

For the measurements of propagation property of the wave, the system shown in Fig. 1 is used. The amplitude of the wave propagated directly from the driving position and the amplitude of the wave propagated after reflecting at the reflector are measured by the pick-up coil, which are indicated as $A_{0}$ and $A_{1}$ in the figure, respectively. The ratio of $A_{1} / A_{0}$ is an attenuation ratio of the wave propagated for a length of $x$, where $x / 2$ is a distance between the pick-up coil and the reflector. The reflection of the wave is performed by clamping the wire by a brass tool. The reflection efficiency as much as 0.8 is obtained by this method, which is discussed in the next section. Although the ratio of $A_{1} / A_{0}$ also includes an loss of the wave at the reflector, the measurement system shown in Fig. 1 is useful for the experiments of the propagation of the wave for a long distance. It is because the

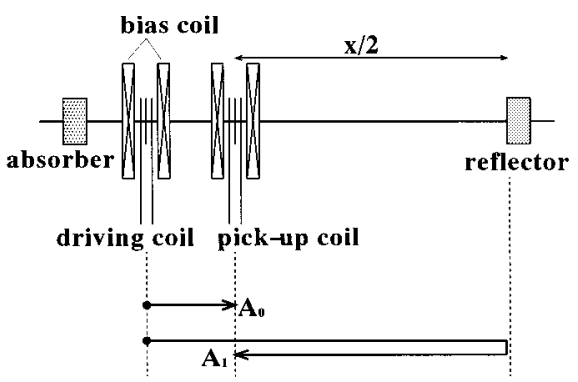

FIG. 1. System for driving and pickup of the magnetoelastic waves and for measuring propagation and reflection properties of the waves. 




FIG. 2. Measured amplitude ratio $A_{1} / A_{0}$ as a function of the propagation length of the magnetoelastic wave. The driving frequency of the wave is varied from $500 \mathrm{kHz}$ to $4 \mathrm{MHz}$.

propagation length can be easily varied by changing the position of the clamp reflector without changing the position of the driving and pick-up coils. This technique is applied for the distance sensor. ${ }^{3}$

Figure 2 shows the measured amplitude ratio $\left(A_{1} / A_{0}\right)$ indicated as a function of the propagation length up to $6 \mathrm{~m}$. The driving frequency was varied from $500 \mathrm{kHz}$ to $4 \mathrm{MHz}$. In the figure, the attenuation constant of the wave is obtained as a slope of the amplitude ratio against the propagation length. The intercept of the amplitude ratio where the propagation length equals to zero indicates a reflection efficiency of the wave at the reflector. With increasing driving frequency, the attenuation of the wave increases for the measured frequency range. It is because eddy current loss increases for a propagation of the high-frequency magnetoelastic wave. The wave driven at $500 \mathrm{kHz}$ is propagated with a low attenuation constant of $0.18 \mathrm{~m}^{-1}$, which corresponds to $8.6 \mathrm{~dB}$ for $10 \mathrm{~m}$. It is expected to be observed with a sufficient-signal intensity after the propagation of 20 or $30 \mathrm{~m}$.

\section{B. Reflection property}

The distance sensor using the magnetoelastic wave measures a distance between the driving/pick-up coil and the reflector by counting the propagation time of the wave. In order to obtain a sufficient signal intensity of the wave propagated through a long distance, an efficient reflection of the wave is required. Figure 3 shows the measured amplitudes of the wave propagated after the reflection $\left(A_{1}\right.$ in Fig. 1 ) in the cases of $x=0.6,2.0$, and $4.0 \mathrm{~m}$, where $x$ is the propagation length indicated in Fig. 1. The driving frequency of the wave is $1 \mathrm{MHz}$. As shown in the insertion in Fig. 3, the sample wire is clamped between pieces of brass material. It is found that the load of $5 \mathrm{~N}$ is required to obtain a sufficient reflection of the wave by the reflector used in this experiment. The frequency dependence of the reflection efficiency is also studied. As for materials of the clamp reflector, acrylic resin, stainless steel, and brass are studied. The load on the clamp is enough to obtain a maximum reflection in the measurement. As shown in Fig. 4, it is found that the reflec-



FIG. 3. Measured amplitudes of the reflected magnetoelastic wave as a function of the load on the clamp reflector. The driving frequency of the wave is $1 \mathrm{MHz}$.The insertion shows the reflector clamping the sample wire.

tion efficiency decreases with increasing the driving frequency of the magnetoelastic wave and that it is as much as 0.9 for the driving frequency below $2 \mathrm{MHz}$. The reflector of stainless steel has a high reflection efficiency in the highfrequency region above $2 \mathrm{MHz}$ compared with that of brass, which is attributed to the large elastic constant of stainless steel. The figure also indicates the reflection efficiency for the acrylic resin reflector. The reflection of the wave is not observed for the driving frequency above $2 \mathrm{MHz}$. It is also found that when a soft urethane rubber is utilized as wave absorber shown in Fig. 1 the reflection efficiency is quite low.

Because the attenuation constant of the wave is small and the reflection efficiency is large for the wave driven at lower frequency, the frequency around $500 \mathrm{kHz}$ is advantageous for applications such as the distance sensor. Although an accuracy of the distance sensor is high for the high driving frequency, the resolution as small as $1 \mathrm{~mm}$, which is practically sufficient, is obtained at $500 \mathrm{kHz}$ in the distance sensor having a detect range of $0-5 \mathrm{~m}$.

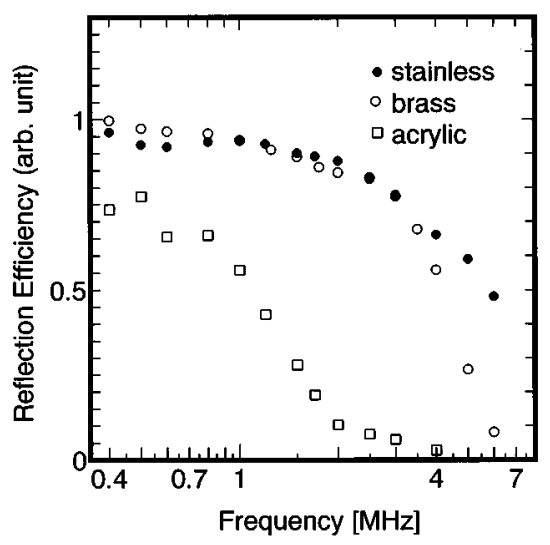

FIG. 4. Reflection efficiency of the magnetoelastic waves as a function of the driving frequency. $(\bigcirc),(\bigcirc)$, and $(\square)$ indicate the results for stainless steel, brass, and acrylic resin used as materials of the clamp, respectively. 


\section{CONCLUSIONS}

Propagation and reflection properties of the magnetoelastic waves in $\mathrm{Fe}_{77.5} \mathrm{Si}_{7.5} \mathrm{~B}_{15}$ amorphous wires are studied. Due to a large magnetostriction of $\mathrm{Fe}_{77.5} \mathrm{Si}_{7.5} \mathrm{~B}_{15}$, efficient driving and pickup are obtained. The attenuation constant of the wave is measured at various driving frequency. With increasing frequency, the attenuation constant increases, which is due to an enhancement of eddy current loss. As the attenuation constant is quite small $\left(0.18 \mathrm{~m}^{-1}\right)$ at $500 \mathrm{kHz}$, the wave is expected to be observed with a sufficient-signal intensity after the propagation of a long distance. The reflection effi- ciency is also investigated. Using a clamp as reflector, the magnetoelastic wave is reflected with the efficiency as much as 0.9 for the wave driven below $2 \mathrm{MHz}$. These fundamental properties of the magnetoelastic wave are utilized to examine the device performance for devices such as the distance sensor.

${ }^{1}$ S. Masuda and K. Kakuno, IEEE Trans. Magn. MAG-26, 1801 (1990).

${ }^{2}$ L. V. Panina, M. Noda, and K. Mohri, J. Appl. Phys. 76, 6198 (1994).

${ }^{3}$ Y. Takemura, S. Masuda, T. Yamada, and K. Kakuno, IEEE Trans. Magn. MAG-31, 3155 (1995).

${ }^{4}$ M. Hagiwara, A. Inoue, and T. Masumoto, Metall. Trans. 13A, 373 (1982). 